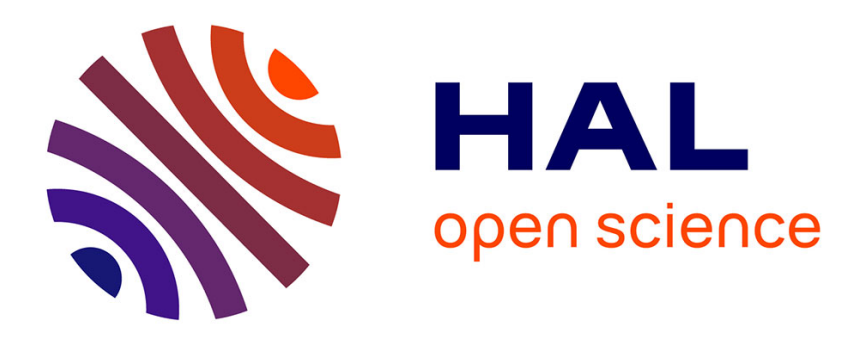

\title{
Noise radiation from doubly periodic reinforced submerged plates
}

\author{
S. Hayek, A. Karali
}

\section{To cite this version:}

S. Hayek, A. Karali. Noise radiation from doubly periodic reinforced submerged plates. Journal de Physique IV Proceedings, 1994, 04 (C5), pp.C5-301-C5-310. 10.1051/jp4:1994562 . jpa-00253057

\section{HAL Id: jpa-00253057 https://hal.science/jpa-00253057}

Submitted on 1 Jan 1994

HAL is a multi-disciplinary open access archive for the deposit and dissemination of scientific research documents, whether they are published or not. The documents may come from teaching and research institutions in France or abroad, or from public or private research centers.
L'archive ouverte pluridisciplinaire HAL, est destinée au dépôt et à la diffusion de documents scientifiques de niveau recherche, publiés ou non, émanant des établissements d'enseignement et de recherche français ou étrangers, des laboratoires publics ou privés. 


\title{
Noise radiation from doubly periodic reinforced submerged plates
}

\author{
S.I. HAYEK ${ }^{(1)}$ and A. KARALI
}

Dept. Engineering Science and Mechanics, Penn State University, University Park, PA 16801, U.S.A.

\begin{abstract}
Almost all known structures are designed for low weight and high strength. For metal structures, especially aircrafts and underwater vehicles, this means reinforcement in the form of ribs. This paper presents an analytic model for the prediction of the acoustic scattered field from such rib-reinforced structures. The geometry is assumed to be that of an infinite elastic plate reinforced with a periodic double array of stiffeners. The plate is assumed to be in contact with a homogeneous and isotropic fluid on one side only, its other side being exposed to vacuum. The plate is modeled using the Mindlin plate theory which includes the effects of shear deformation and rotary inertia, thus allowing the evaluation of the scattered acoustic field at frequencies above the coincidence frequency. The reinforcements, exerting both forces and moments on the plate, are included in the model through their transverse and rotational impedances. A Fourier transform technique is used for evaluating the acoustic scattering of an incident plane acoustic wave by the fluid-loaded, reinforced plate and analytic solutions for the backscattered acoustic field are obtained. Numerical results for a wide range of frequencies are also presented.
\end{abstract}

\section{INTRODUCTION}

The problem of sound generated by a vibrating surface is known as the radiation problem from a surface induced to motion by a set of forces. The other problem of interest in the area of research that became known as sound-structure interaction, is the distortion of an acoustic field due to the presence of an object that reflects or scatters sound waves generated in the acoustic medium. In general, the sound scatterer is not considered rigid and therefore the scattering action of a submerged structure is modified by its elastic response. The problem considered in this work, is the acoustic scattering from an elastic plate in contact with an acoustic fluid and reinforced with an infinite periodic double array of stiffening members.

To the best of the authors' knowledge, the first study of scattering of an incoming acoustic wave by a rib-reinforced elastic plate was presented by Konovalyuk [1]. Konovalyuk based his work on the classical plate theory and attempted a solution for the field scattered by $\mathrm{N}$ arbitrarily located reinforcing ribs. He then proceeded to obtain an approximate solution for the backscattered field due to an infinite number of periodically spaced identical ribs.

More than a decade later, Woolley $[2,3]$ improved on Konovalyuk's solution by using Mindlin's plate theory [4]. He first treated the problem of the acoustic scattering from a submerged plate with one reinforcing rib [2] and then formulated the problem for the case of multiple ribs [3]. In both

(1) Currently Visiting Professor at: Laboratoire Vibrations-Acoustique, INSA-Lyon, Bâtiment 303, 20 Avenue Albert Einstein, 69621 Villeurbanne, France 
cases however, the interacting ribs were modeled using a Bernoulli-Euler beam. This inconsistency in the modeling of the plate and the reinforcing ribs essentially limited Woolley's validity of results to frequencies lower than the frequencies allowed by the Mindlin plate theory. Moreover, when Woolley attempted to obtain the solution for the scattered field due to an infinite number of equally spaced identical ribs, his calculations produced a null field. This was an obviously erroneous result since it meant that a plate without ribs and a plate with ribs would produce the same reflected field. Woolley himself appeared puzzled by this result but ended up attributing it to the 'great symmetry' of the problem. It must be mentioned that Woolley was aware, and did reference in his paper [3], Stepanishen's solution [5] of the backscattered acoustic pressure due to an infinite array of identical, periodically spaced line discontinuities.

Stepanishen's study of the acoustic transmission and scattering characteristics of a plate with line impedance discontinuities was also based on the Mindlin plate theory. The discontinuities were assumed to exert only forces on the plate and were characterized by their translational impedances. Two special cases of non-specular scattering were investigated: First the acoustic scattering from a finite-sized array of discontinuities where the acoustic elastic coupling among the discontinuities was neglected and second the scattering from an infinite-sized array of discontinuities. In the latter case the interactions between the scatterers were included in the analysis and an analytic solution for the scattered pressure was found.

Şeren [6] and Şeren and Hayek [7] developed the analytic models for the prediction of the scattered field from an infinite elastic plate with single and multiple line discontinuities. Their work was also based on Mindlin's plate theory and the ribs were modeled correctly using the Timoshenko beam theory, thus correcting the errors of the Woolley model. The solution for the scattered field was in the form of a Fourier integral which was evaluated by the steepest descent method, a Modified Saddle Point method that led to an asymptotic series solution for the scattered velocity potential, as well as a fast converging numerical integration algorithm.

Of interest to the work presented in this paper are also the studies by Mead [8] and Rumerman [9] on the vibration and wave propagation in ribbed plates, Mace [10] on the sound radiated from a plate with two sets of parallel stiffeners and Burroughs [11] on the acoustic radiation from circular cylinders with two sets of periodic ring supports.

\section{THE MATHEMATICAL MODEL}

The aim of this study is to obtain an analytic solution for the acoustic scattered field due to an infinite array of reinforcements on an infinite elastic plate. The reinforcements consist of two sets of parallel ribs of infinite length, one of which is 'heavy' and the other one 'light'. The spacing of the 'heavy' stiffeners is an integer multiple of the spacing of the 'light' ones, thus creating a periodic double array of reinforcements. Each set of stiffeners is characterized by a different set of force $Z_{f}$ and moment $Z_{m}$ input impedance values.

The mathematical model is developed for the general case of multiple reinforcing ribs which may have different geometric and material parameters. After the development of the mathematical model is completed, analytical solutions are sought for the case of the periodic double array of reinforcements, described above. The geometry and coordinate system are shown in Figure 1 . The cartesian coordinate system is centered on an arbitrary rib with the $y$ axis pointing inward into the page and the $x-z$ plane coinciding with the plane of the page. The wave vector of the incident plane wave lies entirely on the $x-z$ plane, thus rendering the acoustic field invariant along the length of the ribs and the scattering problem 2-D.

The mathematical model for the acoustic scattering from a reinforced elastic plate consists of a system of two coupled differential equations: 


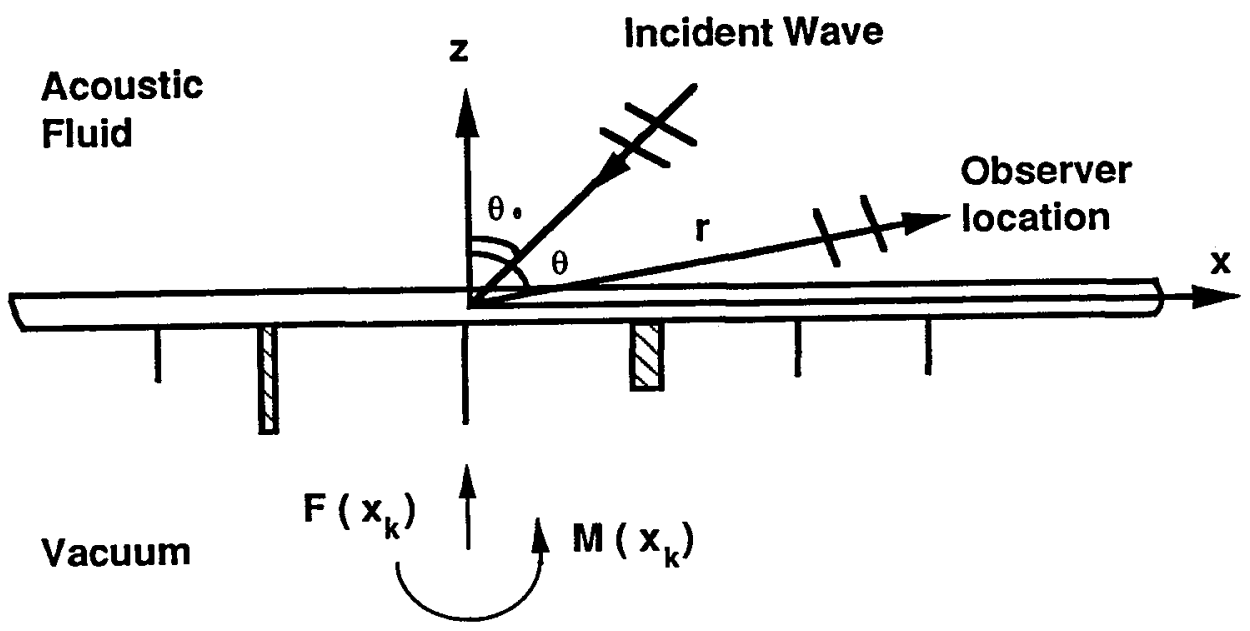

Figure 1: Geometry and Coordinate System of a Plate with Multiple Stiffeners

i) The governing equation of motion of the plate, with the radiation loading and the mechanical forces exerted by the stiffeners included in its forcing function.

ii) The acoustic wave equation, describing the disturbance in the fluid.

The coupling between the elastic plate and the acoustic medium is attained through the continuity condition, which states that the velocity of the upper surface of the plate is equal to the velocity of the acoustic fluid that it is in contact with.

The governing equation of the plate motion is the fourth order differential equation describing the flexural vibrations of elastic plates, derived by Mindlin [4]. Mindlin's plate theory accounts for shear deformation and rotary inertia effects and provides for the two first branches of the frequency spectrum of an elastic plate. In Mindlin's derivation the loads on the surface of the plate are assumed to be normal to the mid-plane of the plate. In our analysis the reinforcements were allowed to exert both forces and moments on the plate and therefore the governing equation of motion of the plate was derived again from first principles.

The governing equation of motion of the fluid-loaded, reinforced elastic plate shown in Figure 1, is

$$
\begin{aligned}
& \frac{\partial^{4} w}{\partial x^{4}}+k_{0}^{2} F_{1} \frac{\partial^{2} w}{\partial x^{2}}+k_{0}^{4} F_{2} w(x)= \\
& \frac{1}{\omega^{2} \rho_{0}}\left[k_{0}^{5} F_{5}-k_{0}^{3} F_{3} \frac{\partial^{2}}{\partial x^{2}}\right] F(x)+\frac{1}{\omega^{2} \rho_{0}} k_{0}^{5} F_{6} \frac{\partial}{\partial x} M(x)
\end{aligned}
$$

where $k_{0}$ is the acoustic wavenumber

$$
k_{0}=\frac{\omega}{c_{0}}
$$

$\omega$ is the circular frequency, $\rho_{0}$ and $c_{0}$ are the mass density and the sound speed in the acoustic fluid, respectively. The coefficients $F_{1}$ through $F_{6}$ are functions of the incident wave frequency, as well as the material and geometric parameters of the plate and the material parameters of the acoustic fluid, 


$$
\begin{aligned}
& F_{1}=\frac{\omega^{2} \rho_{s}}{k_{0}^{2}}\left(\frac{12 D+h^{3} \kappa_{s}^{2} G}{12 \kappa_{s}^{2} G D}\right) \\
& =\left(\frac{24}{\pi^{2}} \frac{1}{1-\nu}+1\right) \frac{1}{\left(c_{p} / c_{0}\right)^{2}} \\
& F_{2}=\frac{\omega^{2} \rho_{s} h}{k_{0}^{4}}\left(\frac{\omega^{2} \rho_{s} h^{2}-12 \kappa_{s}^{2} G}{12 \kappa_{s}^{2} G D}\right) \\
& =\frac{24}{\pi^{2}} \frac{1}{\left(c_{p} / c_{0}\right)^{4}} \frac{1}{1-\nu}+\frac{1}{\Omega^{2}} \\
& F_{3}=\frac{\omega^{2} \rho_{0}}{\kappa_{s}^{2} G h k_{0}^{3}} \\
& =\frac{24}{\pi^{2}} \frac{\rho_{0}}{\rho_{s}} \frac{1}{\left(c_{p} / c_{0}\right)} \frac{1}{(1-\nu)} \frac{1}{\Omega} \\
& F_{5}=\frac{\omega^{2} \rho_{0}}{D k_{0}^{5}}\left(1-\frac{\rho_{s} h^{2} \omega^{2}}{12 \kappa_{s}^{2} G}\right) \\
& =\left(-\frac{4 \sqrt{3}}{\pi^{2}\left(c_{p} / c_{0}\right)^{3}(1-\nu)}+\frac{\left(c_{p} / c_{0}\right)}{2 \sqrt{3} \Omega^{2}}\right) \frac{\rho_{0}}{\rho_{s}} \frac{1}{\Omega} \\
& F_{6}=\frac{\omega^{2} \rho_{0}}{k_{0}^{5} D} \\
& =\frac{\left(c_{p} / c_{0}\right)}{2 \sqrt{3}} \frac{\rho_{0}}{\rho_{s}} \frac{1}{\Omega^{3}}
\end{aligned}
$$

In Equations 3 through 12, $\rho_{s}$ and $h$ are the plate mass density and thickness respectively, $E, G$, $\nu$ are the Young's modulus, shear modulus and Poisson's ratio, $\kappa_{s}^{2}=\pi / 12$ is the Mindlin correction factor, $D=E h^{3} / 12\left(1-\nu^{2}\right)$ is the plate stiffness, $\Omega=\omega / \omega_{c}$ the frequency normalized by the classical plate coincidence frequency $\omega_{c}=\sqrt{12} c_{0}^{2} / h c_{p}$ and $c_{p}=E /\left(1-\nu^{2}\right) \rho_{s}$ is the longitudinal plate sound speed.

The external loads on the plate appearing on the right hand side of Equation 1, may be defined as follows:

$F(x)$ is the sum of the fluid pressure on the upper side of the plate and the mechanical stresses due to the reinforcements

$$
F(x)=-\left.p(x, z)\right|_{z=0}+\left.\sum_{k=-\infty}^{+\infty} Z_{f k} \dot{w}(x)\right|_{x=x_{k}} \delta\left(x-x_{k}\right)
$$

where $Z_{f k}$ is the transverse input impedance of the $k t h$ stiffener, located at $x_{k}$ and $\delta()$ is the Dirac delta function.

$M(x)$ is the sum of the external moments applied on the plate by the reinforcements

$$
M(x)=\left.\sum_{k=-\infty}^{+\infty} Z_{m k} \frac{\partial \dot{w}(x)}{\partial x}\right|_{x=x_{k}} \delta\left(x-x_{k}\right)
$$

where $Z_{m k}$ is the moment input impedance of the $k t h$ stiffener. 
The velocity potential $\Phi(x, z)$ of the acoustic fluid, filling the half-space above the plate, satisfies the homogeneous wave equation

$$
\left(\nabla^{2}+k_{0}^{2}\right) \Phi(x, z)=0
$$

and is related to the fluid pressure $p(x, z)$ by

$$
p(x, z)=\rho_{0} \frac{\partial \Phi}{\partial t}=-i \omega \rho_{0} \Phi(x, z)
$$

In all of the above equations as well as the rest of this paper, the time dependence $\exp (-i \omega t)$ has been dropped for convenience.

The total acoustic field is divided in three parts, the incident field $\Phi_{i}(x, z)$, the reflected field from the plate considered without reinforcements $\Phi_{r}(x, z)$ and the scattered field due to the reinforcements $\Psi(x, z)$,

$$
\Phi(x, z)=\Phi_{i}(x, z)+\Phi_{r}(x, z)+\Psi(x, z)
$$

The incident field is given by

$$
\Phi_{i}(x, z)=\Phi_{0} e^{-i k_{0} \cos \theta_{0} z+i k_{0} \sin \theta_{0} x}
$$

the reflected field from the non-reinforced plate is

$$
\Phi_{r}(x, z)=V\left(\theta_{0}\right) \Phi_{0} e^{i k_{0} \cos \theta_{0} z+i k_{0} \sin \theta_{0} x}
$$

and the scattered field due to the reinforcements is written in the form of a Fourier integral

$$
\Psi(x, z)=\frac{\Phi_{0}\left(1-V\left(\theta_{0}\right)\right)}{\sqrt{2 \pi}} \sum_{j=-\infty}^{+\infty} \int_{-\infty}^{+\infty} A_{j}\left(k_{x}\right) e^{i k_{0} \sin \theta_{0} x_{j}} e^{i\left[k_{x}\left(x-x_{j}\right)+\sqrt{k_{0}^{2}-k_{x}^{2}} z\right]} d k_{x}
$$

In Equation 20, $A_{j}\left(k_{x}\right)$ is the angular spectrum function. In Equation 19, $V\left(\theta_{0}\right)$ is the plane wave reflection coefficient of an air-backed non-reinforced elastic plate and may be written in the following non-dimensional form

with $a\left(\theta_{0}\right)$ and $b\left(\theta_{0}\right)$ given by:

$$
V\left(\theta_{0}\right)=-\frac{a\left(\theta_{0}\right)-i b\left(\theta_{0}\right)}{a\left(\theta_{0}\right)+i b\left(\theta_{0}\right)}
$$

$$
\begin{gathered}
a\left(\theta_{0}\right)=F_{5}+F_{3} \sin \theta_{0}{ }^{2} \\
b\left(\theta_{0}\right)=\left(\sin \theta_{0}{ }^{4}-F_{1} \sin \theta_{0}{ }^{2}+F_{2}\right) \cos \theta_{0}
\end{gathered}
$$

To complete the formulation of the problem, the continuity condition between the plate and the fluid is used

$$
\dot{w}(x)=-\left.\frac{\partial \Phi(x, z)}{\partial z}\right|_{z=0} \quad \text { for } \quad-\infty<x<+\infty
$$

The two differential equations given by Equations 1 and 15 through use of the boundary condition of Equation 24, may be combined to yield a single differential equation for the plate/fiuid system, with one dependent variable, the velocity potential $\Phi(x, z)$

$$
\begin{aligned}
& \left.\frac{\partial^{5} \Phi}{\partial x^{4} \partial z}\right|_{z=0}+\left.k_{0}^{2} F_{1} \frac{\partial^{3} \Phi}{\partial x^{2} \partial z}\right|_{z=0}+\left.k_{0}^{4} F_{2} \frac{\partial \Phi}{\partial z}\right|_{z=0}= \\
& \quad-\left.\left[k_{0}^{5} F_{5}-k_{0}^{3} F_{3} \frac{\partial^{2}}{\partial x^{2}}\right] \Phi(x, z)\right|_{z=0} \\
& \left.\quad \frac{1}{i \omega \rho_{0}}\left[k_{0}^{5} F_{5}-k_{0}^{3} F_{3} \frac{\partial^{2}}{\partial x^{2}}\right] \sum_{k=-\infty}^{+\infty} Z_{f k} \frac{\partial \Phi}{\partial z}\right|_{\substack{x=x_{k} \\
z=0}} \delta\left(x-x_{k}\right) \\
& \left.\quad \frac{1}{i \omega \rho_{0}}\left[k_{0}^{5} F_{6} \frac{\partial}{\partial x}\right] \sum_{k=-\infty}^{+\infty} Z_{m k} \frac{\partial^{2} \Phi}{\partial x \partial z}\right|_{\substack{x=x_{k} \\
z=0}} \delta\left(x-x_{k}\right)
\end{aligned}
$$


The complex Fourier transform and its inverse are defined as

$$
\begin{aligned}
& F\left(k_{x}\right)=\frac{1}{\sqrt{2 \pi}} \int_{-\infty}^{+\infty} f(x) e^{-i k_{x} x} d x \\
& f(x)=\frac{1}{\sqrt{2 \pi}} \int_{-\infty}^{+\infty} F\left(k_{x}\right) e^{+i k_{x} x} d k_{x}
\end{aligned}
$$

If a Fourier transform, defined by Equation 26, is applied to Equation 25, the following expression results for the angular spectrum function $A_{j}(\lambda)$ :

$$
A_{j}(\lambda)=\frac{\bar{C}_{0 j}+\bar{C}_{1 j} \lambda+\bar{C}_{2 j} \lambda}{Q(\lambda)}
$$

where $\lambda=\frac{k_{x}}{k_{0}}$ and $Q(\lambda)$ is the impedance of the fluid loaded plate, given by

$$
Q(\lambda)=\left[\left(F_{5}+F_{3} \lambda^{2}\right)+i \sqrt{1-\lambda^{2}}\left(\lambda^{4}-F_{1} \lambda^{2}+F_{2}\right)\right]
$$

In the general case of a non-periodic array of multiple reinforcements the coefficients $\bar{C}_{0 j}, \bar{C}_{1 j}$ and $\bar{C}_{2 j}$ are obtained as solutions of a $3 N \times 3 N$ system of linear, algebraic equations, where $j=0,1, \ldots, N$.

\section{ANALYTIC SOLUTION OF THE SCATTERED FIELD}

In this section we are focusing our attention to the acoustic scattered field due to an infinite periodic double array of reinforcements. This configuration consists of two sets of reinforcements, characterized by two sets of values of translational $Z_{f}$ and rotational $Z_{m}$ impedances. It is assumed that the stiffeners of the first set, which will be referred to as 'light ribs', are spaced at a distance $d$. The heavier stiffeners, or 'heavy ribs' of the second set, are spaced at a distance $q d$, where $q$ is an integer. Due to the periodicity of the array, the number of coefficients $\bar{C}_{i j}$ required to determine the angular spectrum function, given by Equation 28, reduces to $3 q$. These coefficients are obtained as solutions of the following system of linear, algebraic equations:

$$
\begin{gathered}
\sum_{p=0}^{q-1}\left[\left(\frac{k_{0} F_{5} Z_{f t}}{q d} S_{0 p t}-\delta_{p t} \omega \rho_{0}\right) \bar{C}_{0 p}+\frac{k_{0} F_{5} Z_{f t}}{q d} S_{1 p t} \bar{C}_{1 p}+\frac{k_{0} F_{5} Z_{f t}}{q d} S_{2 p t} \bar{C}_{2 p}\right]= \\
\frac{k_{0}^{2} F_{5} Z_{f t}}{\sqrt{2 \pi}} \cos \theta_{0} \\
\sum_{p=0}^{q-1}\left[\frac{k_{0}^{3} F_{6} Z_{m t}}{q d} S_{1 p t} \bar{C}_{0 p}+\left(\frac{k_{0}^{3} F_{6} Z_{m t}}{q d} S_{2 p t}+\delta_{p t} \omega \rho_{0}\right) \bar{C}_{1 p}+\frac{k_{0}^{3} F_{6} Z_{m t}}{q d} S_{3 p t} \bar{C}_{2 p}\right]= \\
\frac{k_{0}^{4} F_{6} Z_{m t}}{\sqrt{2 \pi}} \cos \theta_{0} \sin \theta_{0} \\
\sum_{p=0}^{q-1}\left[\frac{k_{0} F_{3} Z_{f t}}{q d} S_{0 p t} \bar{C}_{0 p}+\frac{k_{0} F_{3} Z_{f t}}{q d} S_{1 p t} \bar{C}_{1 p}+\left(\frac{k_{0} F_{3} Z_{f t}}{q d} S_{2 p t}-\delta_{p t} \omega \rho_{0}\right) \bar{C}_{2 p}\right]= \\
\frac{k_{0}^{2} F_{3} Z_{f t}}{\sqrt{2 \pi}} \cos \theta_{0}
\end{gathered}
$$

In the above system of equations, $S_{n p t}$ denote the summation

$$
S_{n p t}=\sum_{j=-\infty}^{+\infty} \frac{\lambda_{j}^{n} \sqrt{1-\lambda_{j}^{2}}}{Q\left(\lambda_{j}\right)} e^{i \frac{2 \pi j}{q}(t-p)}
$$


where $t, p=0,1,2, \ldots,(q-1)$ and $n=0,1,2,3, Z_{f t}$ and $Z_{m t}$ are the transverse and moment input impedances of the $t^{t h}$ reinforcement within one period of the double array and $\delta_{p t}$ is Kronecker's delta. Since $t$ ranges from 0 to $q-1$ the dimension of the above system is $3 q \times 3 q$. It should be noted that this system reduces to a $3 \times 3$ system, if either all stiffeners are identical (and therefore characterized by one set of values of force and moment input impedances) or when the impedances of the lighter stiffeners are set equal to zero and $d$ is substituted for the product $q d$. In both cases the array is reduced to an infinite, periodic array of stiffeners with identical material and geometric properties.

In order to obtain and implement the solution for the acoustic scattered field due to a periodic double array of stiffeners, a specific example is considered here. In it, the number of lighter ribs placed within two heavy ribs is two and therefore $q=3$. Equation 30 results in a system of 9 linear algebraic equations in 9 unknowns, $\bar{C}_{0 p}, \bar{C}_{1 p}$ and $\bar{C}_{2 p}$ where $p=b, r 1, r 2$ corresponds to a bulkhead and the first and second rib to the right of the bulkhead, respectively.

The $9 \times 9$ system can be easily solved numerically yielding the values of $\bar{C}_{i p}$. These values are subsequently substituted to the integral form of the scattered field given by Equation 20 . The order of summation and integration is then reversed and Poisson's summation formula employed

$$
\sum_{j=-\infty}^{+\infty} \delta(x-j d)=\frac{1}{d} \sum_{j=-\infty}^{+\infty} e^{-\frac{i 2 \pi j x}{d}}
$$

to yield the analytic solution for the acoustic scattered field, due to a periodic double array of reinforcements:

$$
\begin{aligned}
\Psi(x, z)= & \frac{\sqrt{2 \pi}}{3 k_{0} d} \Phi_{0}\left(1-V\left(\theta_{0}\right)\right) \\
& \times \sum_{j=-\infty}^{+\infty}\left\{A_{b}\left(\lambda_{j}\right)+A_{r 1}\left(\lambda_{j}\right) e^{-\frac{2 \pi j}{3}}+A_{r 2}\left(\lambda_{j}\right) e^{-\frac{4 \pi j}{3}}\right\} e^{i k_{0} \lambda_{j} x} \\
& \times \begin{cases}e^{-k_{0} z} \sqrt{\lambda_{j}^{2}-1} & \text { if } \lambda_{j}^{2}>1 \\
e^{i k_{0} z \sqrt{1-\lambda_{j}^{2}}} & \text { if } \lambda_{j}^{2}<1\end{cases}
\end{aligned}
$$

where

$$
\lambda_{j}=\sin \theta_{0}+\frac{2 \pi j}{3 k_{0} d} \quad-\infty<j<+\infty
$$

and $A_{b}$ is the angular spectrum function of the acoustic field due to the heavy ribs and $A_{r 1}, A_{r 2}$ the angular spectrum functions due to the first and second light ribs to the right of the heavy rib, respectively.

\section{NUMERICAL RESULTS AND DISCUSSION}

Numerical results are presented in this section for the non-specular scattering of an incident plane acoustic wave by a periodically reinforced steel plate loaded with water on one side only. Assuming a lossless plate, the following values of material parameters are used in the numerical calculations:

- Young's modulus $E=1.95 \times 10^{11} \mathrm{~N} / \mathrm{m}^{2}$

- Poisson's ratio $\nu=0.27$

- plate density $\rho_{s}=7.7 \times 10^{3} \mathrm{~kg} / \mathrm{m}^{3}$

- fluid density $\rho_{0}=10^{3} \mathrm{~kg} / \mathrm{m}^{3}$

- sound velocity in water $c_{0}=1460 \mathrm{~m} / \mathrm{s}$ 


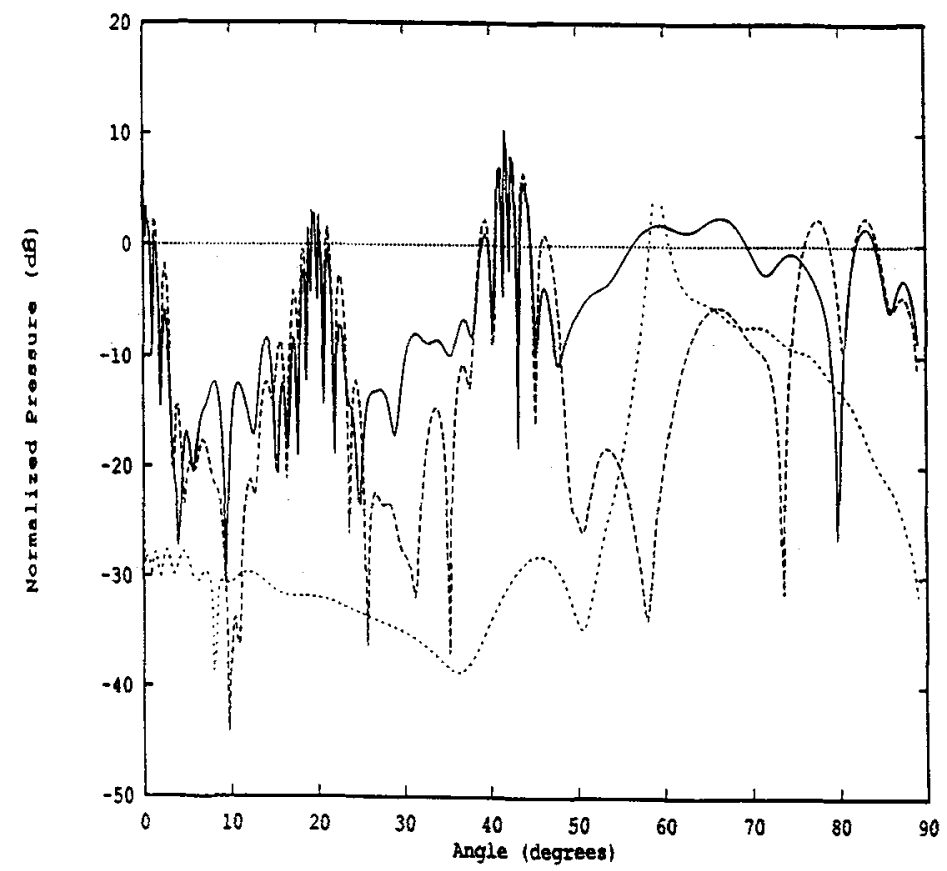

Figure 2: (a) Normalized Backscattered Pressure for $\Omega=1.0$

The impedances of the stiffeners were normalized to the non-dimensional variables $Z_{f}^{*}$ and $Z_{m}^{*}$ given by:

$$
\begin{aligned}
Z_{f}^{*} & =\frac{\omega_{c}}{E} Z_{f} \\
Z_{m}^{*} & =\frac{\omega_{c}^{3}}{E c_{p}^{2}} Z_{m}
\end{aligned}
$$

where the classical coincidence frequency $\omega_{c}$ was defined in Section 2.

The backscattered pressure field due to the infinite array of reinforcements and normalized to the incident plane wave was computed from Equation 33. Figures 2 (a) through (d) show the normalized backscattered field at observer distance $k_{0} r=100$. The geometry chosen, consists of heavy ribs and light ribs placed in such a way, so that the period of the heavy ribs equals $3 d$, where $d$ is the spacing of the light ribs located within one spacing of the heavy ribs. The distance $d$ is set equal to one acoustic wavelength, at the classical coincidence frequency, and the values of the non-dimensional $Z_{f}^{*}$ and $Z_{m}^{*}$ are:

$$
\begin{array}{r}
Z_{f b}^{*}=Z_{m b}^{*}=i \\
Z_{f r}^{*}=Z_{m r}^{*}=i 10^{-2}
\end{array}
$$

where the subscripts $b$ and $r$ indicate heavy ribs and light ribs respectively. The normalized backscattered pressure is plotted as function of the observation angle $\theta$ and for frequencies $\Omega=1.0,4.0,8.0$, 15.0. For comparison purposes, for each frequency $\Omega$, the results for the field due to an infinite array of periodic light ribs, placed at a distance $d$, as well as the field due to the heavy ribs alone, at their spacing $3 d$, are also presented along with the plots for the field due to the periodic double array. 

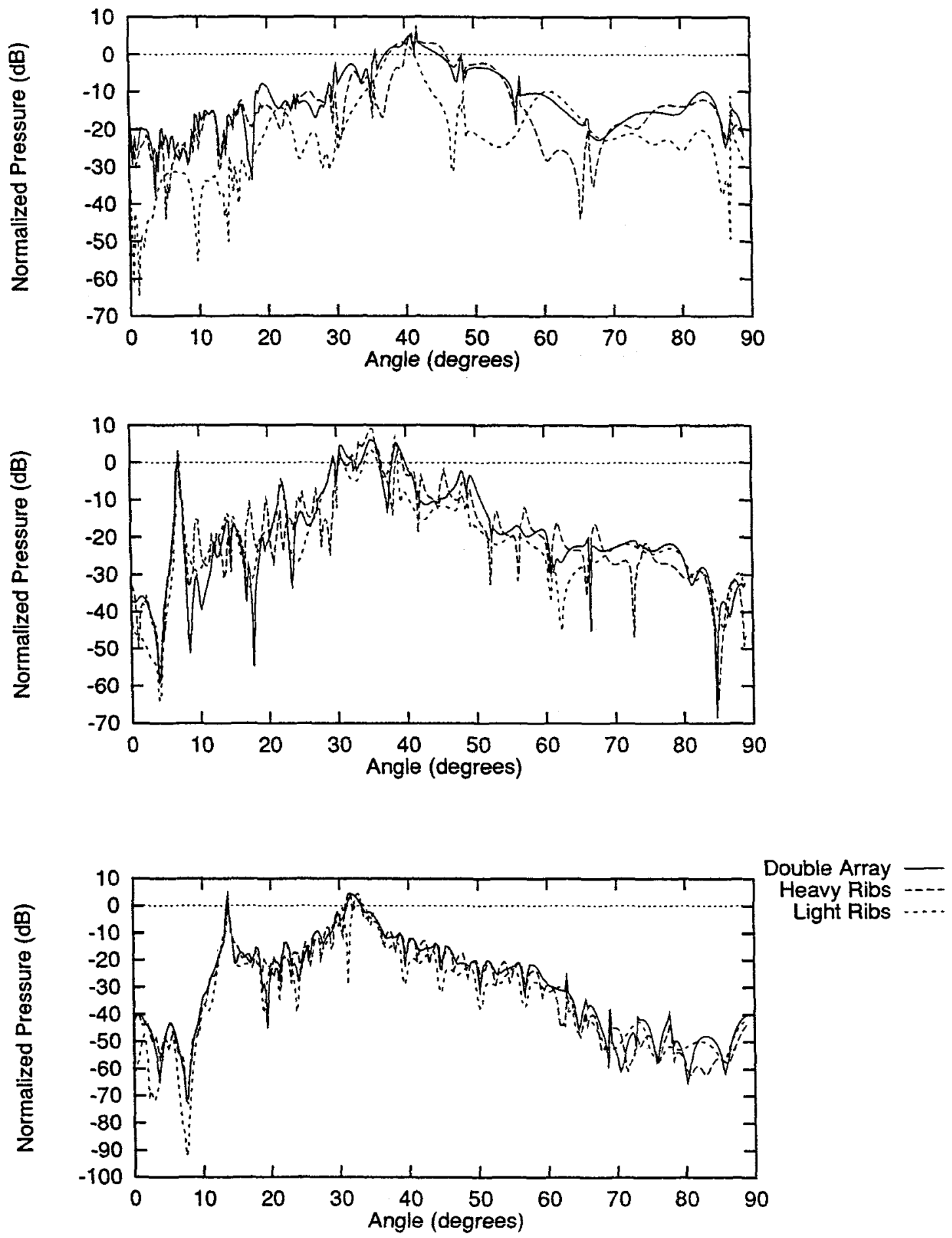

Figures 2(b)-(d): Normalized Backscattered Pressure for $(b) \Omega=4.0,(c) \Omega=8.0,(d) \Omega=15.0$ 
The backscattered field due to an incoming wave of frequency equal to the classical coincidence frequency of the plate is shown in Figure 2 (a). At this frequency the radiation due to the ribs only, is much lower than the radiation caused by the double array, containing both light and heavy ribs. For some observation angles there is as much as 35 to $40 \mathrm{db}$. difference between the two fields, indicating that the scattered pressure from the double array at $\Omega=1.0$ is primarily due to the heavy ribs. This indicates that the non-specular scattered field, at low frequencies, is primarily driven by the impedance values of the reinforcements. As the frequency increases above the Mindlin plate coincidence frequency (which is approximately $20 \%$ higher than the classical coincidence frequency) the radiation pattern begins to show a peak. The peak becomes stronger as $\Omega$ increases. When the frequency exceeds the second coincidence frequency, also known as the cut-off frequency of the Mindlin plate, (Cut-off frequency for a steel plate in water, $\Omega=7.01$ ) a second peak is forming in the radiation pattern, starting near the normal to the plate and slowly shifting to the right, with increasing frequency.

It should also be noted that the separation between all three curves diminishes with increasing frequency and at very high frequencies the field due to the periodic double array, containing heavy and light ribs, may very well be approximated by the field due to the periodic array of the heavy ribs alone.

\section{References}

[1] I.P. Konovalyuk. Diffraction of a plane sound wave by a plate reinforced by stiffness members. Soviet Physics-Acoustics, 14:465-469, 1969.

[2] B.L. Woolley. Acoustic scattering from a submerged plate i. One reinforcing rib. Journal of the Acoustical Society of America, 67:1642-1653, 1980.

[3] B.L. Woolley. Acoustic scattering from a submerged plate ii. Finite number of reinforcing ribs. Journal of the Acoustical Society of America, 67:1654-1658, 1980.

[4] R.D. Mindlin. Influence of rotatory inertia and shear on flexural motions of isotropic, elastic plates. Journal of Applied Mechanics, 18:31-38, 1951.

[5] P.R. Stepanishen. The acoustic transmission and scattering characteristics of a plate with line impedance discontinuities. Journal of Sound and Vibration, 58(2):257-272, 1978.

[6] Çetin Seren. Acoustic Scattering from Ribs-reinforced Plates-Uniform Asymptotic Solution. PhD thesis, The Pennsylvania State University, University Park, PA, 1986.

[7] C. Şeren and S.I. Hayek. Acoustic radiation from an insonified elastic plate with a line discontinuity. Journal of the Acoustical Society of America, 86(1):195-209, 1989.

[8] D.J. Mead. A new method for analyzing wave propagation in periodic structures; Applications to periodic Timoshenko beams and stiffened plates. Journal of Sound and Vibration, 104(1):9-27, 1986.

[9] M.L. Rumerman. Vibration and wave propagation in ribbed plates. Journal of the Acoustical Society of America, 57(2):370-373, 1975.

[10] B.R. Mace. Sound radiation from a plate reinforced by two sets of parallel stiffeners. Journal of Sound and Vibration, 71(3):435-441, 1980.

[11] Courtney B. Burroughs. Acoustic radiation from fluid-loaded infinite circular cylinders with doubly periodic ring supports. Journal of the Acoustical Society of America, 75(3):715-722, 1984. 